\section{Sleep Coaching for Sleep Inversion in Smith-Magenis Syndrome}

An 18-month-old girl with genetically confirmed SmithMagenis syndrome (SMS) presented to the pediatric sleep clinic with excessive behavioral problems and a poor sleep pattern. She would start feeling drowsy between 5-6 PM, followed by multiple awakenings lasting 5-8 minutes, requiring being bottle fed or rocked. She would wake up at $2 \mathrm{AM}$ and remain active and playful thereafter. She was hyperactive and restless throughout the day, associated with temper tantrums and head banging. A 24-hour polysomnography showed decrease in total sleep time ( 6 hours), delayed sleep latency (22 min), delayed REM latency (132 min) and multiple night awakenings. There were no features of obstructive sleep apnea.

Sleep coaching was initiated by setting a regular sleep routine at night. A time gap of one hour between feeding or play and sleep was maintained. All sleep associations in the form of rocking and feeding as well as co-sleeping were stopped with graduated extinction. Her night time sleep was delayed by 15 minutes each day, till she was able to sleep by 9 PM. The bed room was darkened and all access to multimedia screens was removed. Within one month, she was able to sleep by 9 PM and wake up at 6:30 AM, with no night awakenings. Her behavioral symptoms and tantrums during the day resolved. She was maintaining this schedule at the 6-month follow-up.

SMS is characterized by infantile hypotonia, expressive speech delay, mental retardation, short stature, scoliosis, characteristic craniofacial features and self-injurious behavior
[1]. Sleep issues in children with SMS commonly include early sleep onset, frequent nocturnal awakenings, early morning arousal and daytime sleepiness [2]. There is increasing evidence of an inverted melatonin rhythm in SMS with low levels of melatonin at night, and significantly high levels during the day [3]. Behavioral problems increase in children when the levels peak and sleep attacks are noted when levels drop. Administering melatonin; however, only enables a patient to sleep earlier and does not affect the early morning awakening or behavioral changes. Sleep coaching has shown to produce reliable and durable changes in infant sleep patterns [4]. This report demonstrates that sleep issues in children with SMS can be managed with sleep coaching alone.

KR BHARATH KUMAR REDDY From Department of Paediatric Pulmonology and Sleep, Shishuka Children's Hospital, Bangalore, Karnataka, India.

\section{REFERENCES} drbharathreddykr@gmail.com

1. Greenberg F, Lewis RA, Potocki L, et al. Multidisciplinary clinical study of Smith-Magenis syndrome (deletion 17p11.2). Am J Med Genet. 1996;62:247-54.

2. De Leersnyder H, De Blois MC, Claustrat B, et al. Inversion of the circadian rhythm of melatonin in the SmithMagenis syndrome. J Pediatr. 2001;139:111-6.

3. Potocki L, Glaze D, Tan DX, et al. Circadian rhythm abnormalities of melatonin in Smith-Magenis syndrome. J Med Genet. 2000;37:428-33.

4. Mindell JA, Kuhn B, Lewin DS, Meltzer LJ, Sadeh A. Behavioural treatment of bedtime problems and night wakings in infants and young children. Sleep. 2006; 29:1263-76.

\section{Repetitive Eye Poking in an Infant - A Diagnostic Conundrum}

Poor vision in a growing child can affect all domains of development [1]. Early diagnosis of poor vision in infants is extremely challenging as they are unable to express sensory loss or cooperate for clinical and equipment-based testing [2]. We report one such infant who presented with repetitive eye poking.

A 1-year-old female child born out of a second degree consanguineous marriage presented with complaints of not picking up objects, looking downwards without making eye contact, and with repetitive eye poking since three months of life. There was no history of head or ocular injury and no family history of visual impairment. The infant was born at term following an uneventful antenatal period. Infant's growth and development were appropriate for age. Infant had photophobia, bilateral nystagmus and sluggish pupillary reflex but was able to fix and follow light at a distance of $30 \mathrm{~cm}$. Slit lamp examination revealed normal anterior segment and no evidence of pigmentary retinopathy on fundus examination. Differential diagnosis considered at this point were optic nerve hypoplasia, Leber's congenital amaurosis (LCA), high refractory errors, early onset rod-cone dystrophy, achromatopsia and cortical visual impairment. Both scotopic and photopic electroretinogram (ERG) were unrecordable. Brain MRI did not show any abnormality. In view of poor vision since birth, nystagmus, normal development, normal fundus, normal brain imaging and flat ERG, a provisional diagnosis of LCA was made. Clinical exome sequencing showed a homozygous missence variation in exon 9 of $G U C Y 2 D$ gene 